\title{
Active regions on the Sun with high flare productivity and strong geomagnetic efficiency
}

\author{
G.A. Porfir'eva, G.V. Yakunina, and A.B. Delone \\ Sternberg Astronomical Institute, Moscow State University, Universitetskij Prospect 13, \\ Moscow 119992, Russia email:yakunina@sai.msu.ru
}

\begin{abstract}
Relations between the geomagnetic $\mathrm{A}_{p}$-index and magnetic and flare parameters of the super active regions (SARs) are analysed. The small and mean SARs having the spot areas $\mathrm{S} \leqslant 1000 \mu h$ seem to differ from the SARs being large $\delta$-spots (SARs- $\delta$ ) not only by their parameters but also by relations between them.
\end{abstract}

\section{Introduction}

Investigations of flare prolific and geomagnetic effective active regions (ARs) on the Sun help us to understand better solar sources of space weather and Earth magnetic field disturbances. More than two dozens of super active regions, that produced major solar flares and severe geomagnetic storms during last several decades, are examined. Among them there were chosen ten SARs with an unusual morphology being large $\delta$ spots: NOAA 5395, 5629, 5698, 5747, 6555, 6659, 6891, 7321, 9077 and 9393 . At least by two from their three parameters of the activity such as the X-ray flare index XRI, proton flux $\mathrm{F}_{p}$ and geomagnetic $\mathrm{A}_{p}$ index the $\mathrm{SARs}-\delta$ stand in the first dozen among all ARs.

\section{Data and analysis}

The ARs with $\delta$-configuration have been investigated repeatedly and observed on the coude-refractor Opton with the $\mathrm{H}_{\alpha}$-filter at High Altitude Station by Sternberg Institute. The data about the flares and other characteristics were taken from Solar Geophysical Data (SGD), web-sites at http://www.sec.noaa.gov and http://umbra.nascom.nasa.gov/SEP/, Catalogue of solar proton events, 1987 - 1998, Moscow, 1998 and published papers.

Solar and geomagnetic activity of the SARs is presented by the maximal sunspots area $\mathrm{S}, \mathrm{XRI}, 10.7$ radio and proton fluxes $\mathrm{F}$ and geomagnetic index $\mathrm{A}_{p}$. Tilt angle $\varphi$ between the AR magnetic axis and solar equator and force-free parameter $\alpha$, demonstrating correspondingly the AR general orientation relatively to E-W direction and magnetic field non-potentiality and twist are considered as the ARs magnetic properties.

Almost by all their characteristics the SARs being large $\delta$-spots stand out among other SARs. They have the sun spots areas $\mathrm{S} \geqslant 1000 \mu \mathrm{h}, \mathrm{XRI} \geqslant 20$ and $\mathrm{F}(10.7) \geqslant 1000$ sfu. Many of them produce $\mathrm{A}_{p} \geqslant 150$ and $\mathrm{F}_{p} \geqslant 1000 \mathrm{pfu}$. The small and mean SARs, having $\mathrm{S} \leqslant 1000 \mu \mathrm{h}$, such as NOAA 5800, 6703, 7154, 7671, 9026, 9236, 9415 and some others, produce generally $\mathrm{XRI} \leqslant 10$ and $\mathrm{A}_{p} \leqslant 150$ although many of them give $\mathrm{A}_{p} \geqslant 50$. The relationship between the tilt angle $\varphi$ and mean helicity $\alpha$ for the $\operatorname{SARs}-\delta$ is shown in Figure 1 (left). The values of $\varphi$ and $\alpha$ are taken from Tian, Liu \& Wang (2002), but the sign of $\varphi$ corresponds to the definition proposed by Tian et al. (2001). A letter S or $\mathrm{N}$ after the AR index number indicates the position of the AR in the southern or northern hemisphere. All the SARs besides SAR 5747 have the normal $\alpha$-sign. On the 

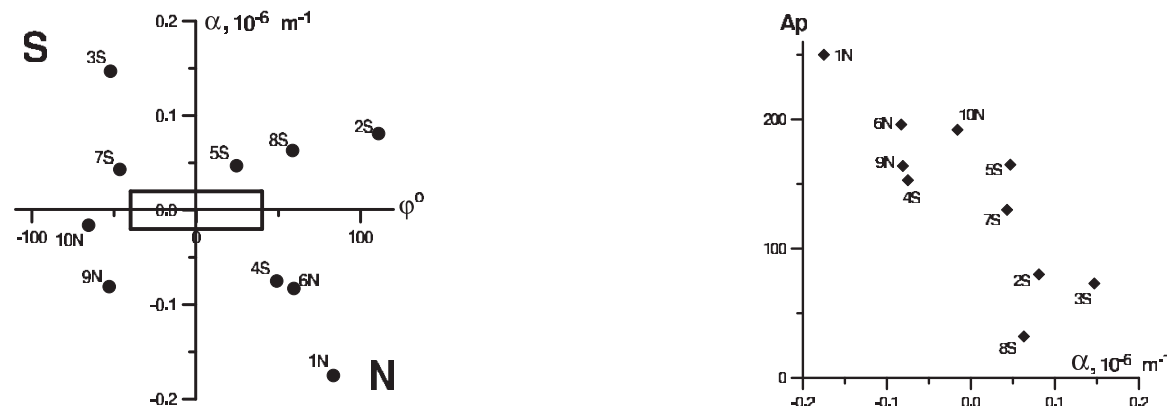

Figure 1. Left: Relationship between the tilt angle $\varphi$ and the mean helicity $\alpha$ for the SARs- $\delta$, NOAA: 1S-5395, 2S-5629, 3S-5698, 4S-5747, 5S-6555，6N-6659，7S-6891, 8S-7321, 9N-9077, $10 \mathrm{~N}-9393$. The rectangle outlines the area inside of which the $\varphi$ and $\alpha$ values are for usual ARs: $|\varphi| \leqslant 40^{\circ}$ (Tian et al. 2001) and $|\alpha| \leqslant|\bar{\alpha} \pm 3 \sigma|$ (Pevtsov, Canfield \& Metcalf 1995). The quadrants for the normal ARs are marked by "S" and "N" symbols. Right: Relation between $\mathrm{A}_{p}$-index and average helicity $\alpha$ for the SARs- $\delta$.

contrary only four SARs- $\delta$ have the tilt angles of the normal sign (but no values) and normal chirality. The rest SARs- $\delta$ have no normal sign of the tilt angle and consequently no normal chirality. Both the $\alpha$ and tilt for the SAR 5747 are abnormal.

For the SARs $\delta$-spots the $\mathrm{A}_{p}$-index is correlated with the total magnetic flux, tilt angle and $\alpha$ and increases generally with an increase of XRI, S and F(10.7). On the one hand it is known that magnetic twist increases the possibility of flares arising. On the other hand the $\mathrm{A}_{p}$ decreases when $\alpha$ increases as it can be seen from the Figure 1 (right) and a north-south asymmetry exists. But as it was demonstrated by Pevtsov, Canfield \& Metcalf (1994) flares occur when changes in the $\alpha$ are observed in ARs. The small and mean SARs having $\mathrm{S} \leqslant 1000 \mu \mathrm{h}$ seem to differ from the SARs large $\delta$-spots not only by their parameters but also by the relations between them. Specifically the tilt angle appears not so essential for the small SARs as for the SARs large $\delta$-spots. The $\mathrm{A}_{p}$-index increases with $\mathrm{S}$ for the SARs- $\delta$ but decreases for the small and mean SARs.

\section{Summary}

Only $30-40 \%$ of the SARs $\delta$-spots obey to the Hale-Nicholson law on the contrary to the typical ARs with no or a low activity, more than $70 \%$ of which follow this law. According to different observations about $60 \%$ of the typical ARs have normal chirality adhering to the helicity sign rule and Hale-Nicholson's law. Their tilt angles and mean twist helicities have opposite handedness. The SARs being the large $\delta$-spots seem mainly or not to adhere to the hemispheric helicity sign rule or not to follow law by Hale-Nicholson or not to obey to both of them at once. Therefore about 50-60\% of them have no normal chirality and their tilt and twist helicity are often of the same handedness. The abnormal chirality of the SARs- $\delta$ seems to be one of the causes of their super activity.

\section{References}

Pevtsov, A.A., Canfield, R.C. \& Metcalf, T.R. 1994 Aph.J. 425, L117-L119.

Pevtsov, A.A., Canfield, R.C. \& Metcalf, T.R. 1995 Aph.J. 440, L109-L112.

Tian, L., Bao, S., Zhang, H. \& Wang, H. 2001 A \& $A$ 374, 294-300.

Tian,L., Liu, Y. \& Wang J. 2002 Solar Phys. 209, 361-374. 